\title{
Transposição monocular vertical dos músculos retos horizontais em pacientes esotrópicos portadores de anisotropia em A
}

\author{
Monocular vertical displacement of the horizontal rectus muscles in esotropic patients \\ with "A"pattern
}

\author{
Ana Carolina Toledo Dias ${ }^{1}$ \\ Mauro Goldchmit ${ }^{2}$ \\ Carlos Ramos de Souza Dias ${ }^{3}$ \\ Frederico Augusto Costa Reis ${ }^{4}$
}

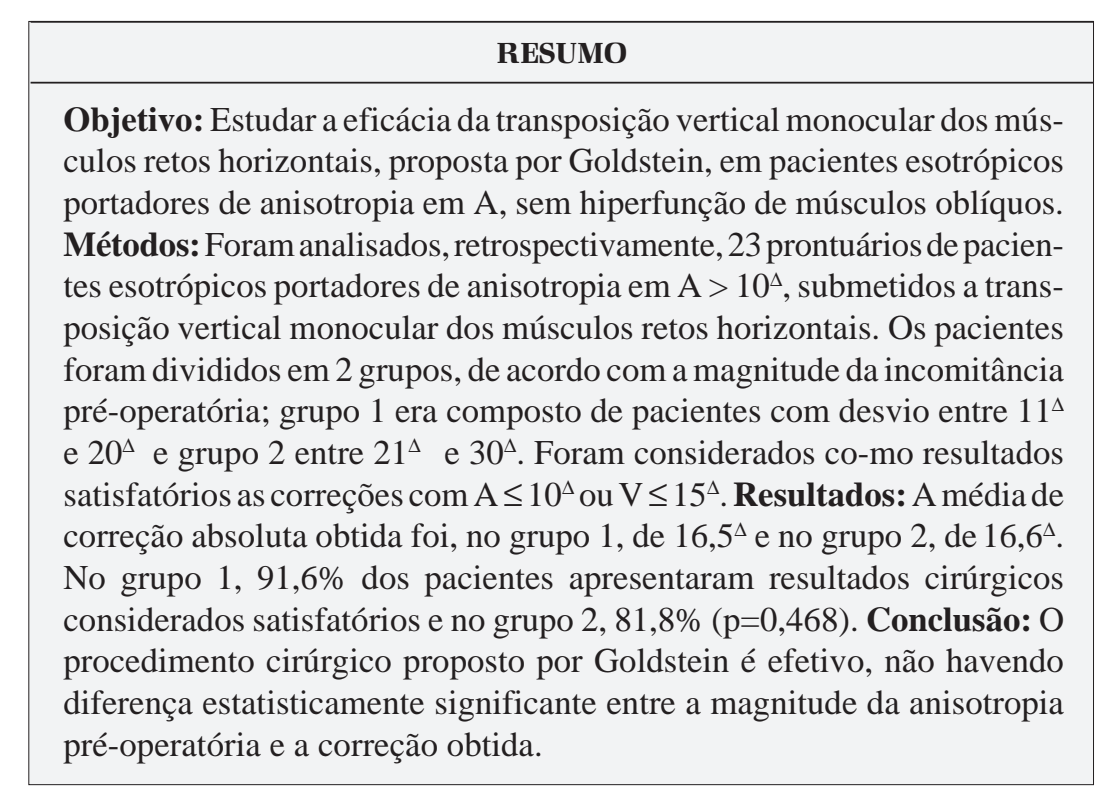

Descritores: Estrabismo; Esotropia/cirurgia; Músculos oculomotores; Movimentos oculares

\section{INTRODUĈ̃̃O}

A etiologia das anisotropias do meridiano vertical tem sido assunto controverso desde a década de 50, tendo surgido a partir de então diversas teorias a esse respeito ${ }^{(1-4)}$. Atualmente, parece unânime atribuir-se o problema, na maioria dos casos, à disfunção dos músculos oblíquos, quer seja de causa inervacional ou mecânica ${ }^{(1,4)}$.

Entretanto, algumas vezes a magnitude da incomitância é desproporcional ao grau de disfunção desses músculos, o que faz supor a existência de algum outro fator como causa. Recentemente, propuseram que a heterotopia das polias dos músculos retos possa ser a causa dessas incomitâncias ${ }^{(5)}$.

Há, ainda, casos de anisotropia sem disfunção de músculos oblíquos e hiperfunção destes sem anisotropia ${ }^{(6)}$.

$\mathrm{O}$ tratamento das anisotropias em A e V difere de acordo com a presença ou ausência de hiperfunção de músculos oblíquos associada. Nos casos em que ela existe, o tratamento cirúrgico é feito através do debilitamento desses músculos ${ }^{(1,7-11)}$. Quando não existe disfunção ou esta é discreta e desproporcional à magnitude da incomitância, preconiza-se a transposição vertical dos músculos retos horizontais, monocular ou binocular ${ }^{(6-9)}$. 
Goldstein $^{(12)}$, em 1967, propôs a transposição vertical monocular de 4 a 8 milímetros (mm) dos músculos retos horizontais, em um grupo de 17 pacientes amblíopes, que apresentavam anisotropia média em A de 15 dioptrias $\left(^{\Delta}\right)$ e em $\mathrm{V}$ de $25^{\Delta}$. O deslocamento realizado seguiu a recomendação de $\mathrm{Knapp}^{(13)}$, em que os músculos são transpostos no sentido em que se deseja reduzir sua ação.

Dos pacientes com anisotropia em A, 6 apresentavam exotropia (XT) e 3 esotropia (ET) e, dos pacientes com anisotropia em V, 5 apresentavam XT e 3 ET. Dentre os pacientes esotrópicos com anisotropia em A, um apresentava anisotropia pré-operatória de $15^{\Delta}$ e a transposição dos músculos retos horizontais realizada foi de $5 \mathrm{~mm}$, com correção de $5^{\Delta}$. Nos dois pacientes em que a transposição foi de $8 \mathrm{~mm}$, um apresentava anisotropia pré-operatória de $16^{\Delta}$ e a correção foi de $24^{\Delta}$ e, no outro, com anisotropia pré-operatória de $25^{\Delta}$, a correção obtida foi de $40^{\Delta}$ (ambos transformaram-se em anisotropias em V de $8^{\Delta}$ e $15^{\Delta}$ respectivamente).

Este trabalho teve como objetivo estudar a eficácia da transposição vertical monocular dos músculos retos horizontais, proposta por Goldstein ${ }^{(12)}$, na correção de anisotropia em A em pacientes esotrópicos, tratados na Seção de Motilidade Extrínseca Ocular da Clínica Oftalmológica da Santa Casa de Misericórdia de São Paulo.

\section{MÉTODOS}

Foi realizado estudo retrospectivo de 23 prontuários de pacientes [com esotropia média na posição primária do olhar (PPO) de $35,52^{\Delta} \pm 14,12^{\Delta}\left(20^{\Delta}\right.$ a $\left.70^{\Delta}\right)$ ], portadores de anisotropia do meridiano vertical em $\mathrm{A}$, sem hiperfunção de músculos oblíquos, submetidos à cirurgia de transposição monocular de $5 \mathrm{~mm}$ dos músculos retos horizontais, segundo a técnica proposta por Goldstein ${ }^{(12)}$ (elevação do músculo reto medial e abaixamento do músculo reto lateral). Para a correção da esotropia foram submetidos a retrocesso do músculo reto medial em média de $5 \mathrm{~mm} \pm 1,5 \mathrm{~mm}$ (4 mm a $11 \mathrm{~mm}$ ) e ressecção do músculo reto lateral em média de $6,5 \mathrm{~mm} \pm 9 \mathrm{~mm}(5 \mathrm{~mm}$ a $8 \mathrm{~mm})$. Todos os prontuários pertencem à Seção de Motilidade Extrínseca Ocular da Clínica Oftalmológica da Santa Casa de Misericórdia de São Paulo e as operações foram realizadas entre 01/06/1977 e 27/07/2001, pelos assistentes da Seção, obedecendo a técnica padronizada (sutura direta do músculo transposto na esclera).

A amostra de pacientes foi composta por 9 homens (39\%) e 14 mulheres (61\%), com idade média de $15 \pm 8,6$ anos (4 a 37).

Todos os pacientes eram amblíopes (diferença de 2 ou mais linhas de acuidade visual com a melhor correção óptica, na tabela de Snellen) e não apresentavam incomitância longe/ perto. Realizou-se medida dos desvios em supra e infraversão máximas, através de "cover test" alternado com prismas ou pelo método de Krimsky, de acordo com a colaboração do paciente e a severidade da ambliopia.

As cirurgias foram realizadas sempre no olho com menor acuidade visual. O período pós-operatório mínimo para avaliação do resultado foi de 3 meses, com média de 5,3 \pm 4,0 meses.

Considerou-se anisotropia em $\mathrm{A}$ as variações do desvio horizontal, entre a supra e a infraversão, maiores que $10^{\Delta}$. Foram considerados como sucesso cirúrgico os resultados pós-operatórios com anisotropia em $\mathrm{A} \leq 10^{\Delta}$ e em $\mathrm{V} \leq 15^{\Delta}$.

Os pacientes foram divididos em 2 grupos, de acordo com a magnitude da incomitância pré-operatória, sendo o primeiro grupo formado pelos pacientes portadores de anisotropia entre $11^{\Delta}$ e $20^{\Delta}(\mathrm{N}=12)$ e o segundo entre $21^{\Delta}$ e $30^{\Delta}(\mathrm{N}=11)$.

Para análise dos resultados, foram aplicados os seguintes testes: teste exato de Fisher, com a finalidade de estudar a associação entre sucesso cirúrgico pós-operatório nos grupos estudados; teste de Wilcoxon, para comparar os valores dióptricos pré e pós-operatórios em cada um dos grupos e teste de Mann-Whitney, para comparar as correções obtidas entre os grupos 1 e 2. Fixou-se em 0,05 ou 5\% o nível de rejeição da hipótese de nulidade.

\section{RESULTADOS}

Os resultados encontram-se nas tabelas 1 e 2 .

\section{DISCUSÃO}

Almeida empregou a cirurgia monocular dos músculos retos horizontais associada ao seu deslocamento vertical de 4 a $5 \mathrm{~mm}$ em 23 pacientes esotrópicos com anisotropias em A e $\mathrm{V}^{(14)}$. Vinte pacientes portavam anisotropia em $\mathrm{V}$ e 3 em $\mathrm{A}$. A menor correção obtida foi de $10^{\Delta}$ e a maior de $54^{\Delta}$, com média de $25^{\Delta}$. No presente estudo, observou-se que não houve diferença estatisticamente significante entre os valores de correção absoluta encontrados em ambos os grupos analisados (p>0,05) (Tabela 1). A correção média no grupo 1 foi de 16,5 , enquanto no grupo 2 foi de $16,6^{\Delta}$ (Tabela 1 ); portanto, não houve correlação entre o tamanho da anisotropia pré-operatória e a correção obtida. Esses resultados diferem dos de Almei$\mathrm{da}^{(14)}$, que observou que a magnitude da correção é proporcional à magnitude da anisotropia pré-operatória, pois os grandes desvios tiveram correções maiores que os pequenos.

Teoricamente, essa cirurgia pode produzir efeito torcional sobre o olho operado, com possível diplopia pós-operatória, o que não foi demonstrado por Almeida ${ }^{(14)}$. Todos os pacientes submetidos a este procedimento, analisados no presente estudo, eram amblíopes, para que se evitasse essa possível complicação.

Não houve diferença estatisticamente significante $(\mathrm{p}=$ 0,468 ) entre os resultados considerados satisfatórios em ambos os grupos, ou seja, anisotropia pós-operatória em $\mathrm{A} \leq 10^{\Delta}$ ou em $\mathrm{V} \leq 15^{\Delta}$. No grupo $1,91,6 \%$ dos pacientes obtiveram correção da anisotropia e, no grupo 2,81,8\% (Tabela 2).

A transposição vertical de ambos os músculos retos mediais de $5 \mathrm{~mm}$, em 37 pacientes esotrópicos portadores de 
anisotropia em A, com desvios pré-operatórios entre $10^{\Delta}$ e $24^{\Delta}$, foi efetiva, apresentando melhores resultados os com incomitâncias menores que $20^{\Delta(8)}$. Houve relação inversamente proporcional entre magnitude da anisotropia pré-operatória e bom resultado cirúrgico $\left(\mathrm{A}<10^{\Delta} \mathrm{e} \mathrm{V}<15^{\Delta}\right)$, fato não observado no presente estudo. Observando-se os resultados apresentados na tabela 1, no grupo de menor incomitância préoperatória $\left(11^{\Delta}\right.$ a $\left.20^{\Delta}\right), 3$ pacientes ficaram subcorrigidos (25\%) e no grupo com anisotropia pré-operatória maior $\left(21^{\Delta}\right.$ a $\left.30^{\Delta}\right)$ a subcorreção ocorreu em 7 pacientes $(63,6 \%)$. Essa informação é importante para os pacientes portadores de esotropia e que apresentam exotropia pós-operatória em infraversão, desvio que com o tempo pode desestabilizar o resultado obtido na PPO. Com os resultados obtidos, acredita-se que, frente a incomitâncias verticais maiores que $20^{\Delta}$, a transposição vertical monocular dos músculos retos horizontais deva ser maior que $5 \mathrm{~mm}$. Dessa forma, analisando-se os grupos individualmente, percebe-se que a eficácia do procedimento cirúrgico

\begin{tabular}{|c|c|c|c|c|c|}
\hline \multicolumn{3}{|c|}{ Grupo 1} & \multicolumn{3}{|c|}{ Grupo 2} \\
\hline Pré & Pós & Correção & Pré & Pós & Correção \\
\hline 11 & 12 & -1 & 22 & 0 & 22 \\
\hline 15 & -5 & 20 & 22 & 10 & 12 \\
\hline 15 & -6 & 21 & 22 & 10 & 12 \\
\hline 15 & 0 & 15 & 23 & 0 & 23 \\
\hline 15 & 0 & 15 & 23 & 0 & 23 \\
\hline 15 & 4 & 11 & 25 & 10 & 15 \\
\hline 15 & -15 & 30 & 25 & 25 & 0 \\
\hline 15 & 0 & 15 & 25 & 2 & 23 \\
\hline 20 & 0 & 20 & 25 & 0 & 25 \\
\hline 20 & 8 & 12 & 30 & 22 & 8 \\
\hline 20 & 0 & 20 & 30 & 10 & 20 \\
\hline 20 & 0 & 20 & & & \\
\hline$M=16,3$ & & 16,5 & 24,7 & & 16,6 \\
\hline \multicolumn{6}{|c|}{$\begin{array}{l}\text { Fonte: Setor de Motilidade Extrínseca Ocular da Santa Casa de Misericórdia } \\
\text { de São Paulo, } 2002 \\
\text { Teste de Mann-Whitney (correção grupo } 1 \text { x grupo 2) p p }>0,05 \\
\text { O sinal -, na coluna "Pós", significa transformação da anisotropia em A para } \\
\text { V e na coluna "Correção", aumento da forma em A; M= média }\end{array}$} \\
\hline
\end{tabular}

Tabela 2. Pacientes esotrópicos portadores de anisotropia em A, submetidos a transposição vertical monocular dos músculos retos horizontais nos grupos $1\left(11^{\Delta}\right.$ a $\left.20^{\Delta}\right)$ e $2\left(21^{\Delta}\right.$ a $\left.30^{\Delta}\right)$, segundo 0 resultado cirúrgico

$\begin{array}{lccc} & \text { Grupo 1 } & \text { Grupo 2 } & \text { Total } \\ \text { Bom } & 11(91,6 \%) & 9(81,8 \%) & 20(86,9 \%) \\ \text { Mau } & 1(8,4 \%) & 2(18,2 \%) & 3(13,1 \%) \\ \text { Total } & 12 & 11 & 23\end{array}$

Fonte: Setor de Motilidade Extrínseca Ocular da Santa Casa de Misericórdia de São Paulo, 2002

Testes exatos de Fisher, $p=0,468$

Bom - $A \leq 10^{\Delta}$ ou $V \leq 15^{\wedge}$. Mau $-A>10^{\Delta}$ ou $V>15^{\Delta}$ diminui à medida que aumenta a magnitude do desvio préoperatório. Contudo, é necessário maior número de pacientes, para que se comprove esta afirmação estatisticamente.

Em outro estudo, comparou-se os resultados entre a cirurgia monocular e a binocular para correção de anisotropias em $\mathrm{A}$ e $\mathrm{V}^{(14)}$. Os pacientes por ele estudados foram divididos em dois grupos, o primeiro constituído de 28 pacientes com esotropia com desvios, na PPO entre $20^{\Delta}$ e $60^{\Delta}$ sendo que 4 apresentavam anisotropia em A entre $20^{\Delta}$ e $40^{\Delta}$. O segundo grupo era composto por 25 pacientes esotrópicos com desvios na PPO entre $20^{\Delta}$ e $60^{\Delta}$, sendo 10 casos com anisotropia em A, que variavam entre $20^{\Delta} \mathrm{e} 40^{\Delta}$. Os pacientes do primeiro grupo foram submetidos à transposição vertical monocular dos músculos retos horizontais, enquanto, no segundo grupo, a transposição binocular dos músculos retos horizontais. Concluiu, assim, que ambas as técnicas foram eficazes na correção das incomitâncias, sendo a média de correção obtida com a cirurgia monocular $\left(29,7^{\Delta}\right)$ moderadamente superior à média de correção obtida com a cirurgia binocular $\left(17,5^{\Delta}\right)$ e que no primeiro grupo a correção foi proporcional ao grau de anisotropia préexistente, corroborando a afirmação anterior, mesmo com resultados tidos como satisfatórios em ambos os grupos; pacientes com incomitâncias menores obtiveram desvios pósoperatórios menores.

Para a correção da esotropia os pacientes foram submetidos a retrocesso do músculo reto medial em média de $5 \mathrm{~mm}$ (4 mm a $11 \mathrm{~mm}$ ) e ressecção do músculo reto lateral em média de $6,5 \mathrm{~mm}$ (5 mm a $8 \mathrm{~mm}$ ). É possível, sob o ponto de vista mecânico, supor-se que o efeito da transposição vertical dos músculos retos horizontais possa ser variável dependendo da magnitude do retrocesso do músculo reto medial realizado. $\mathrm{Na}$ amostra, em 22 dos 23 pacientes estudados os retrocessos do músculo reto medial variaram de 4 a $6 \mathrm{~mm}$, diferença que a nosso ver provavelmente não deve interferir no resultado da transposição. Seguramente futuros estudos serão necessários para investigar uma possível variação do efeito da transposição com diferentes magnitudes dos retrocessos, essa análise não foi realizada por não se tratar do objetivo deste estudo.

\section{CONCLUSÃO}

Conclui-se assim que, nos pacientes esotrópicos portadores de anisotropia em A com incomitâncias entre $11^{\Delta}$ e $30^{\Delta}$, a transposição monocular vertical dos músculos retos horizontais de $5 \mathrm{~mm}$ é efetiva, não havendo diferença estatisticamente significante entre a magnitude da anisotropia pré-operatória e a correção obtida.

\section{ABSTRACT}

Purpose: To report the effectiveness of the vertical monocular displacement of the horizontal rectus muscles, proposed by Goldstein, in esotropic patients with A pattern, without oblique muscle overaction. Methods: A retrospective study 
was performed using the charts of 23 esotropic patients with A pattern $>10^{\Delta}$, submitted to vertical monocular displacement of the horizontal rectus muscles. The patients were divided into 2 groups in agreement with the magnitude of the preoperative deviation, group $1\left(11^{\Delta}\right.$ to $\left.20^{\Delta}\right)$ and group $2\left(21^{\Delta}\right.$ to $\left.30^{\Delta}\right)$. Satisfactory results were considered when corrections A $\leq 10^{\Delta}$ or $\mathrm{V} \leq 15^{\Delta}$ were obtained. Results: The average of absolute correction was, in group $1,16.5^{\Delta}$ and, in group $2,16.6^{\Delta}$. In group $1,91.6 \%$ of the patients presented satisfactory surgical results and in group 2, 81.8\% ( $\mathrm{p}=0.468)$. Conclusion: The surgical procedure, proposed by Goldstein, is effective and there was no statistical difference between the magnitude of the preoperative anisotropia and the obtained correction.

Keywords: Strabismus; Esotropia/surgery; Oculomotor muscles; Eye movements

\section{REFERÊNCIAS}

1. Urrets-Zavalía A, Solares-Zamora J, Olmos HR. Anthropological studies on the nature of cyclovertical squint. Br J Ophthalmol. 1961;45:578.
2. Urist M. The etiology of the so-called A and V syndromes. Am J Ophthalmol. 1958;46(6):835-44.

3. Bielschowsky A. Lectures on motor anomalies. Am J Ophthalmol. 1938;21: 843. Jampolsky A. The A and V syndromes. Strabismus. In: Symposium of Ophthalmology. Transactions of the New Orleans Academy Ophthalmology. St. Louis: Mosby; 1962. p.157.

4. Clark RA, Muller JM, Rosenbaum AL, Demer JL. Heterotopic muscle pulleys or oblique muscle disfunction? J AAPOS. 1998;2(1):17-25.

5. Souza-Dias CR, Prieto-Diaz J. Anisotropias em A e V. In: Prieto-Diaz J, Souza-Dias CR. Estrabismo, $4^{\mathrm{a}}$ ed. São Paulo: Santos; 2002. p.247-65.

6. Golstein JH. Pitfalls in the diagnosis and treatment of the A and V patterns Int Ophthalmol Clin. 1971;11(2):57-79.

7. Garrido JT, Goldchmit M, Souza-Dias CR. Correção da anisotropia em A em pacientes esotrópicos mediante transposição vertical dos retos mediais. In: $14^{\circ}$ Congresso Latinoamericano de Estrabismo. Anais. São Paulo; 2000. p.373-7.

8. Fink W. The A and V syndromes. Am Orthopt J. 1959;9:105-10.

9. Costenbader F. Symposium: the A and V patterns in strabismus. Trans Am Acad Ophthalmol Otolaryngol. 1964;68:385-6,

10. Pullman MD, Goldstein JH. Effect of monocular vertical displacement on the deviation in primary position. Arch Ophthalmol. 1980;98(11):2001-3.

11. Goldstein JH. Monocular vertical displacement of the horizontal rectus muscles in the A and V patterns. Am J Ophthalmol. 1967;64(4):265-7.

12. Knapp P. Vertically incomitant horizontal strabismus: The so called A and V syndromes. Trans Am Ophthalmol Soc. 1959;57:666-99.

13. Almeida HC. Correction of $\mathrm{A}$ and $\mathrm{V}$ syndromes acting upon only one eye. In: $2^{\text {nd }}$ Meeting of the International Strabismological Association. Proceedings. 134-7,1974

14. Almeida HC. Correção cirúrgica das anisotropias em A e V [tese]. Belo Horizonte: Universidade Federal de Minas Gerais; 1978.

\section{Simpósio Internacional do Hospifal Oftalmológico de Sorocaba}

\section{Curso de Imersão em Transplante de Córnea}

\section{5 a 6 de Novembro de 2004 Sorocabc - SP}

INFORMAC̣ÕES: Tel: (15) 212-9077 (Srta. Aline)

E-mail: ceo@hosbos.com.br

Home-page: www.hosbos.com.br 